\title{
Study of various intrauterine pathologies by hysteroscopy leading to various gynaecological problems
}

\author{
Vandana Agarwal ${ }^{1}$, Rekha Mehani ${ }^{2 *}$ \\ ${ }^{1}$ Department of Obstetrics and Gynaecology, RKDF MCH RC Bhopal, Madhya Pradesh, India \\ ${ }^{2}$ Department of Pharmacology, RKDF MCH RC Bhopal, Madhya Pradesh, India
}

Received: 04 December 2020

Accepted: 29 December 2020

*Correspondence:

Dr. Rekha Mehani,

E-mail: drrekhasanjay@gmail.com

Copyright: $\odot$ the author(s), publisher and licensee Medip Academy. This is an open-access article distributed under the terms of the Creative Commons Attribution Non-Commercial License, which permits unrestricted non-commercial use, distribution, and reproduction in any medium, provided the original work is properly cited.

\section{ABSTRACT}

Background: This study was to diagnose different types of intrauterine pathologies, their locations and relations with clinical presentations (like abnormal uterine bleeding (AUB), recurrent abortions, unexplained infertility and secondary dysmenorrhoea) by hysteroscopy. To know the prevalence and identification of the demographic relations of different intrauterine pathologies and clinical symptoms were observed. This study also aimed in selecting OPD or indoor operative procedure.

Methods: Data collection of 144 cases was done with suspicion of intrauterine pathology on the basis of history and clinical examination. They were screened by per abdomen, per speculum, pelvic examination. USG and HSG in cases of infertility were used. Further intrauterine pathologies were explored by hysteroscopy

Results: On hysteroscopy no abnormality was detected in 80/144 (55.6\%) cases which was the maximum finding observed. AUB 96 (66.7\%) was the most common presenting complaint followed by infertility 39 (27.1\%). In 96 cases there was no organic pathology. Thickened endometrium was the commonest 17/144 (11.8\%) pathology observed by hysteroscopy followed by endometrial polyp 14/144 (9.7\%).

Conclusions: With increasing demands for one-stop clinics where diagnosis and treatment are offered in same sitting. Diagnostic hysteroscopy by virtue of its diagnostic and therapeutic capabilities can become the procedure of first choice in evaluation of gynaecological problems.

Keywords: Hysteroscopy, Pathologies, AUB, Infertility, Recurrent abortions

\section{INTRODUCTION}

Diagnosis and treatment of various gynecological problems have progressed markedly in the light of evidence-based practice and accurate diagnosis. Failure to diagnose and manage any gynecological problem at the appropriate time may lead to serious and chronic consequences. Diagnosis of intrauterine pathologies of patients attending gynecology OPD presenting mainly with complaints of abnormal uterine bleeding, recurrent abortions, infertility and secondary dysmenorrhea depends on the clinical presentations, internal pelvic examination and investigations. Although history and physical examination provide the foundation, a contemporary safe, and comprehensive evaluation of all possible causes, appropriate and immediate therapy can be initiated either by newly developed medical strategies or through minimally invasive surgeries. With improvement in endoscopic equipment and development of accurate and feasible hysteroscopy, we can diagnose and treat various intrauterine pathologies leading to gynecological problems. ${ }^{1}$ Endometrial morphology like normal endometrium, atrophic endometrium and endometrial hyperplasia can be visualized. Structural uterine anomalies such as arcuate uterus, septate/subseptate uterus and bicornuate uterus can be seen. Uterine abnormalities like submucous myomas, small polyp, cornual blockage and uterine synechiae can be effectively diagnosed by hysteroscopy. Hysteroscopy 
is a safe and well-tolerated procedure where diagnosis and treatment are offered in same sitting.

With improvements in endoscopic equipment and development of accurate and feasible hysteroscopy, the increasing demands for one-stop clinic where investigations and treatment are offered simultaneously, in cases of abnormal uterine bleeding, suspected Müllerian anomalies, removal of foreign bodies, and when abnormal imaging findings need to be confirmed..$^{2,3}$

\section{METHODS}

This study was conducted in the department of obstetrics and gynaecology at RKDF medical college and research centre Bhopal, Madhya Pradesh. It is prospective, crosssectional descriptive, hospital-based study from March 2018 to September 2020 in women attending gynae OPD.

Data collection of 144 cases was done. Endometrial hyperplasia, Submucous fibroid, retained products of conception, misplaced IUCD, pre-malignancy, intra uterine adhesions, Submucous myoma, polyp, septate uterus, bicornuate uterus, developmental defects of uterus, cornual block were included pathologies. Patients with abnormal vaginal bleed, recurrent pregnancy loss, Infertility and secondary dysmenorrhoea were included in the study. Patients with profuse uterine bleed with or without haemodynamic instability, pregnancy or immediate post-partum, severe pelvic inflammatory disease, overt genital malignancy, cervical stenosis and pubertal and young unmarried girls were included. Patients were selected on the basis of complaints. They were screened by per abdomen, per speculum, pelvic examination, USG and HSG in cases of infertility were used. Further intrauterine pathologies were explored by hysteroscopy. Data collection was done of all patients with suspicion of intrauterine pathology by history and clinical examination. Eligible patients were properly counselled and informed consent obtained before including into the study. Each patient's age, parity, age at menarche, menstrual history, along with documentation of hypertension, diabetes, obesity, use of any drug or hormones, any previous surgery or treatment was recorded. Routine baseline investigations of blood, urine and USG were done for all patients and special investigations like HSG was done in selected cases. After informed consent and routine preoperative preparation, hysteroscopy was performed in the operation theatre under short general anaesthesia. Using rigid $5 \mathrm{~mm}$ hysteroscope with double flow sheath and $30^{\circ}$ fore oblique view (pro-MIS CE 0123), the procedure was carried out. Systematic inspection of endocervical canal, internal OS endometrium, uterine cavity and tubal ostia was carried out and findings were recorded.

Data analysis was done by SPSS system " $p$ " value using the chi square test was taken out which was found to be significant. There were no ethical, legal or financial aspects involved as test was mere extension of diagnostic protocol employed in the evaluation of gynaecological problems.

\section{RESULTS}

Hysteroscopic findings were normal in 80/144. (55.6\%) which was the commonest finding in our study among abnormal findings $64(50.55 \%)$. Thickened endometrium was the commonest 17/144 (11.8\%). Followed by endometrial polyp 14/144 (9.7\%). Submucous fibroid 11/144 (7.6\%). Amongst infertility group maximum changes comprised of visualization of single ostia 10/144 (6.9\%). 1 case with misplaced cu-T was seen. Youngest patient was of 20 years. Oldest patient was of 54 years. Maximum number of patients $51(35.4 \%)$ were of the age group 31-40 years. Mean age was-37 years. Maximum number of patients were nullipara 38/144 (26.4\%). These were mainly cases of infertility. Next group was of parity 3 35/144 (24.3\%). These were mainly cases of AUB in reproductive age group. In our study maximum cases were of AUB $96(66.7 \%)$. Next were the case of infertility $39(27.1 \%)$.

Table 1: Hysteroscopic findings in participants.

\begin{tabular}{|lll|}
\hline Findings & Participants & Percentages (\%) \\
\hline Normal & 80 & 50.55 \\
\hline Pathologies & 64 & 44.44 \\
\hline Total & 144 & 100 \\
\hline
\end{tabular}

Table 2: Distribution of age of participants.

\begin{tabular}{|lll|}
\hline Age (year) & Frequency & Percentage $(\%)$ \\
\hline $\mathbf{2 1 - 3 0}$ & 45 & 31.3 \\
\hline $\mathbf{3 1 - 4 0}$ & 51 & 35.4 \\
\hline $\mathbf{4 1 - 5 0}$ & 44 & 30.6 \\
\hline $\mathbf{5 1 - 6 0}$ & 4 & 2.8 \\
\hline Total & 144 & 100 \\
\hline
\end{tabular}

Table 3: Distribution of parity of participants.

\begin{tabular}{|lll|}
\hline Parity & Frequency & Percent $(\%)$ \\
\hline Nullipara & 38 & 26.4 \\
\hline P1 & 12 & 8.3 \\
\hline P2 & 24 & 16.7 \\
\hline P3 & 35 & 24.3 \\
\hline P4 & 22 & 15.3 \\
\hline P5 & 8 & 5.6 \\
\hline P6 & 5 & 3.5 \\
\hline Total & 144 & 100 \\
\hline
\end{tabular}

Table 4: Distribution of symptoms of participants.

\begin{tabular}{|lll|}
\hline Symptom & Frequency & Percent $(\%)$ \\
\hline AUB & 96 & 66.7 \\
\hline Infertility & 39 & 27.1 \\
\hline Dysmenorrhoea & 5 & 3.5 \\
\hline Recurrent abortion & 4 & 2.8 \\
\hline Total & 144 & 100 \\
\hline
\end{tabular}


Table 5: Distribution of hysteroscopic findings of participants.

\begin{tabular}{|lll|}
\hline Hysteroscopic findings & Frequency & Percent $(\%)$ \\
\hline NAD & 80 & 55.6 \\
\hline Thickened endometrium & 17 & 11.8 \\
\hline Endometrial polyp & 14 & 9.7 \\
\hline Submucous fibroid & 11 & 7.6 \\
\hline Ostia and cavity changes & 10 & 6.9 \\
\hline Partial septa & 4 & 2.8 \\
\hline Endometrial thickening and submucous fibroid & 2 & 1.4 \\
\hline Endometrial thickening and polyp & 1 & 0.7 \\
\hline Cervical polyp & 1 & 0.7 \\
\hline Patchy endometrium & 2 & 1.4 \\
\hline Misplaced cu-t & 1 & 0.7 \\
\hline Adhesions & 1 & 0.7 \\
\hline Total & 144 & 100 \\
\hline
\end{tabular}

Table 6: Distribution of hysteroscopic finding according to age of participants.

\begin{tabular}{|llllll|}
\hline & Age (year) & & & & \\
\hline Hysteroscopic finding & $\mathbf{2 1 - 3 0}$ & $\mathbf{3 1 - 4 0}$ & $\mathbf{4 1 - 5 0}$ & $\mathbf{5 1 - 6 0}$ & Total \\
\hline NAD & 25 & 34 & 20 & 1 & 80 \\
\hline Thickened endometrium & 2 & 8 & 6 & 1 & 17 \\
\hline Endometrial polyp & 3 & 2 & 7 & 2 & 14 \\
\hline Submucous fibroid & 0 & 3 & 8 & 0 & 11 \\
\hline Ostia and cavity changes & 7 & 3 & 0 & 0 & 10 \\
\hline Partial septa & 4 & 0 & 0 & 0 & 4 \\
\hline Endometrial thickening and & 0 & 0 & 2 & 0 & 2 \\
submucous fibroid & 0 & 1 & 0 & 0 & 1 \\
\hline Endometrial thickening and polyp & 0 & 0 & 1 & 0 & 1 \\
\hline Cervical polyp & 2 & 0 & 0 & 0 & 2 \\
\hline Patchy endometrium & 1 & 0 & 0 & 0 & 1 \\
\hline Misplaced cu-t & 1 & 0 & 0 & 0 & 1 \\
\hline Adhesions & 45 & 51 & 44 & 4 & 144 \\
\hline Total & & & & 1 \\
\hline
\end{tabular}

Chi square $=61.46, \mathrm{p}$ value $=0.002$.

Table 7: Distribution of hysteroscopic findings according to symptoms of participants.

\begin{tabular}{|c|c|c|c|c|c|}
\hline \multirow[b]{2}{*}{ Hysteroscopic findings } & \multicolumn{4}{|c|}{ Symptom } & \multirow[b]{2}{*}{ Total } \\
\hline & AUB & Dysmenorrhoea & Infertility & $\begin{array}{l}\text { Recurrent } \\
\text { abortion }\end{array}$ & \\
\hline NAD & 51 & 4 & 25 & 0 & 80 \\
\hline Patchy endometrium & 0 & 0 & 2 & 0 & 2 \\
\hline Misplaced cu -t & 1 & 0 & 0 & 0 & 1 \\
\hline Adhesions & 0 & 0 & 1 & 0 & 1 \\
\hline Thickened endometrium & 15 & 0 & 2 & 0 & 17 \\
\hline Endometrial polyp & 14 & 0 & 0 & 0 & 14 \\
\hline Submucous fibroid & 11 & 0 & 0 & 0 & 11 \\
\hline Ostia and cavity changes & 0 & 1 & 9 & 0 & 10 \\
\hline Partial septa & 0 & 0 & 0 & 4 & 4 \\
\hline $\begin{array}{l}\text { Endometrial thickening and } \\
\text { submucous fibroid }\end{array}$ & 2 & 0 & 0 & 0 & 2 \\
\hline Endometrial thickening and polyp & 1 & 0 & 0 & 0 & 1 \\
\hline Cervical polyp & 1 & 0 & 0 & 0 & 1 \\
\hline
\end{tabular}


In this study our patients were divided into 4 age groups 21-30 years, 31-40 years, 41-50 years, 51-60 years. Maximum patients were in age groups 31-40 years, $51 / 144(35.4 \%)$, maximum pathologies were of thickened endometrium $8 / 51$ cases $(15.68 \%)$ second were in the age group 21-30 years, 45/144 (31.25\%), in this age group maximum pathologies were found as ostial and cavity changes which comprised of 7/45 (15.55\%), above 40 years of age endometrial polyp was the commonest pathology observed.

Maximum cases were of AUB 96/144 (66.66\%) 51/96 $(53.125 \%)$ no abnormality detected. So, DUB cases were ruled out which could be treated medically.45/96 $(46.87 \%)$ had pathologies. As follows thickened endometrium pathologies 15/45 (33.33\%). Endometrial polyp $14 / 45(31.11 \%)$ Submucous fibroid 11/45 (24.44\%). In infertility cases maximum pathologies were of ostia and cavity 9/39 (23.07\%) In recurrent abortion cases $4 / 4(100 \%)$ had intra uterine septal defects in dysmenorrhoea $1 / 5(20 \%)$ had ostia and cavity changes.

\section{DISCUSSION}

AUB is the single most common reason for many gynaecological referrals. ${ }^{4,5}$ The incidence of which is 30$40 \%$ of all gynaecological cases. AUB largely affects the women of reproductive and perimenopausal age group. In most of the cases there is no underlying organic pathology. Thickened endometrium and polyps are the commonest structural lesion in patient of AUB. Though dysfunctional uterine bleeding and benign pathologies occur in younger patients, hyperplasia, atrophy and polyp are commonly seen in older age group. There is very high possibility of missing endometrial diseases when the patients with thickened endometrial curetting are subjected for endometrial sampling after scanning with transvaginal scan for evaluating endometrial diseases. Because of its suitability for out-patient investigation, and its higher diagnostic accuracy hysteroscopy is increasingly replacing endometrial biopsy for the evaluation of AUB. ${ }^{6}$ Hysteroscopy offers a valuable extension of the gynaecologist's armamentarium. Because of its higher diagnostic accuracy, it permits better treatment of various uterine diseases. After hysteroscopy, the elective surgery of the patient can be planned better. Hysteroscopy allows targeted localisation of intrauterine lesions, and is a superior method as compared to blunt curettage to ensure excision of such lesions diagnostic hysteroscopy has highest sensitivity for detection for endometrial hyperplasia, submucosal fibroid and polyp, most common structural lesions behind AUB. ${ }^{7-8}$ Given the fact that AUB largely affects the women of reproductive and perimenopausal age group diagnostic hysteroscopy by virtue of its high efficacy can prevent many hysterectomies in these young women. Hysteroscopy is also becoming an integral part of the infertility work-up. ${ }^{9}$ Hysteroscopy is the gold standard procedure for uterine cavity exploration. ${ }^{10,11}$
Small intrauterine lesions, which may be of great significance in causing reproductive failure (polyps, such as adhesions, or submucous myomas), are diagnosed much more precisely by hysteroscopy. These lesions many times are missed by HSG or even by transvaginal ultrasonography. ${ }^{12,13}$ With increasing demands for onestop clinics where diagnosis and treatment are offered in same sitting. Diagnostic hysteroscopy by virtue of its diagnostic and therapeutic capabilities can be procedure of first choice in evaluation of gynaecological problems.

\section{Limitations}

The only limitation of this study was that it was a single centre trial study.

\section{CONCLUSION}

In the above study we concluded that AUB is the single most common reason for gynaecological referrals and the incidence is $30-40 \%$ of all gynaecological cases.

\section{Funding: No funding sources \\ Conflict of interest: None declared}

Ethical approval: The study was approved by the Institutional Ethics Committee

\section{REFERENCES}

1. Schwarzler $\mathrm{P}$, Concin $\mathrm{H}$, Bosch $\mathrm{H}$, Berlinger A, Wohlgenannt K, Collins WP et al. Outpatient hysteroscopy. Contemp. Rev Obstet Gynecol. 1998;10:295-302.

2. Campo R, Van Belle Y, Rombauts L, Brosens I, Gordts S. Office mini-hysteroscopy. Hum Reprod Update. 1999;5:73-81.

3. Cooper JM, Brady RM. Hysteroscopy in the management of abnormal uterine bleeding. Obstet Gynecol Clin North Am. 1999;26:217-36.

4. Gimpelson RJ, Rappold HO. A comparative study between) An evaluation of sonohysterography and diagnostic hysteroscopy for the assessment of intrauterine pathology. Ultrasound Obstet Gynecol. 1988;158(3 Pt 1):489-92.

5. Menon MKK, Devi PK, Rao KB. Postgraduate Obstetrics and Gynecology. India: Orient Longman. $1982 ; 253$.

6. Wieser F, Kurz C, Wenzl R, Albrecht A, Huber JC, Nagele F. Atraumatic cervical passage at outpatient hysteroscopy. Fertil Steril. 1998;69:549-51.

7. Brooks PG, Serden SP. Hysteroscopic findings after unsuccessful dilatation and curettage for abnormal uterine bleeding. 1988;158(6 Pt 1):1354-7

8. Nagele F, O'Connor H, Davies A, Badawy A, Mohammed H, Magos AL. 2500 outpatient diagnostic hysteroscopies. Obstet Gynecol. 1996;88(1):87-92.

9. Shushan A, Rojansky N. Should hysteroscopy be a part of the basic infertility workup Hum. Reprod. 1999;14:1923-4. 
10. Golan A, Eilat E, Ron-El R, A Herman, Y Soffer, I Bukovsky. Hysteroscopy is superior to hysterosalpingography in infertility investigation. Acta Obstet Gynecol Scand. 1996;75:654-6.

11. Golan A, Eilat E, Ron-El R, Herman A, Soffer Y, Bukovsky I. Hysteroscopy is superior to hysterosalpingography in infertility investigation. Acta Obstetricia et Gynecologica Scandinavica. 1996;75(7):654-6.

12. Prevedourakis C, loutradis D, Kalindis C, Makris N, Aravantinos D. Hysterosalpingography and hysteroscopies in female infertility. Human reprod. 1994;9(12):2353-5.
13. Vercellini P, Cortesi I, Oldani S, De Giorgi O, Crosignani PG. The role of transvaginal ultrasonography and outpatient diagnostic hysteroscopy in the evaluation of patients with menorrhagia. Hum. Reprod. 1997;12:1768-71.

Cite this article as: Agarwal V, Mehani R. Study of various intrauterine pathologies by hysteroscopy leading to various gynaecological problems. Int J Reprod Contracept Obstet Gynecol 2021;10:627-31. 